Note

\title{
Continuum radiative heat transfer modeling in media consisting of optically distinct components in the limit of geometrical optics
}

\author{
W. Lipiński ${ }^{\text {a,* }}$, D. Keene ${ }^{\text {a }}$, S. Haussener ${ }^{\text {b }}$, J. Petrasch ${ }^{c}$ \\ a Department of Mechanical Engineering, University of Minnesota, Minneapolis, MN 55455, USA \\ ${ }^{\mathrm{b}}$ Department of Mechanical and Process Engineering, ETH Zurich, 8092 Zurich, Switzerland \\ c Department of Mechanical and Aerospace Engineering, University of Florida, Gainesville, FL 32601, USA
}

\section{A R T I C L E I N F O}

\section{Article history:}

Received 15 April 2010

Received in revised form

28 June 2010

Accepted 29 June 2010

Keywords:

Radiation

Volume averaging

Continuum modeling

Multi-component

Multi-phase

\begin{abstract}
A B S T R A C T
Continuum-scale equations of radiative transfer and corresponding boundary conditions are derived for a general case of a multi-component medium consisting of arbitrary-type, non-isothermal and non-uniform components in the limit of geometrical optics. The link between the discrete and continuum scales is established by volume averaging of the discrete-scale equations of radiative transfer by applying the spatial averaging theorem. Precise definitions of the continuum-scale radiative properties are formulated while accounting for the radiative interactions between the components at their interfaces. Possible applications and simplifications of the presented general equations are discussed.
\end{abstract}

Published by Elsevier Ltd.

\section{Introduction}

Radiative transfer in media consisting of optically distinct components is encountered in multiple fields of science and engineering including chemical processing, combustion, nuclear and civil engineering, atmospheric sciences, and solar technology. ${ }^{1}$ An important subset are media consisting of components in the range of geometrical optics [1]. They find applications as reacting packed beds, porous heat exchangers, radiant absorbers and burners, and insulating materials. Porous structures used in high-temperature solar thermal and thermo-chemical processes to generate power and produce chemicals are of special interest. Radiative transfer in such materials is

\footnotetext{
* Corresponding author. Tel.: +1612 626 0875; fax: +16126261854

E-mail address: lipinski@umn.edu (W. Lipiński).

${ }^{1}$ The term component refers to a medium constituent having distinct optical and radiative properties at the macroscopic level and it may consist of further constituents that are distinguishable at the microscopic level.
}

often predicted by using continuum models employing appropriate continuum-scale radiative properties [2,3]. ${ }^{2}$ In many engineering applications it is sufficient to determine the radiative properties prior to the solution of the radiative transfer equations (RTEs) for selected medium composition and morphology. This in turn leads to significant reduction of the overall computational time as compared to complete discrete-scale radiative transfer simulations for problems where the RTEs are solved repeatedly.

Previous pertinent studies on continuum-scale radiative properties of multi-component media consisting of individual components in the limit of geometrical optics include determination of the properties for media consisting of an optically thin (non-participating) component and an opaque component [4-8], and for

\footnotetext{
${ }^{2}$ The term continuum scale refers to length scales for which continuum approach to radiative transfer in multi-component media is valid. The term discrete scale refers to length scales associated with single elements of porous structures (particles, pores, struts).
} 


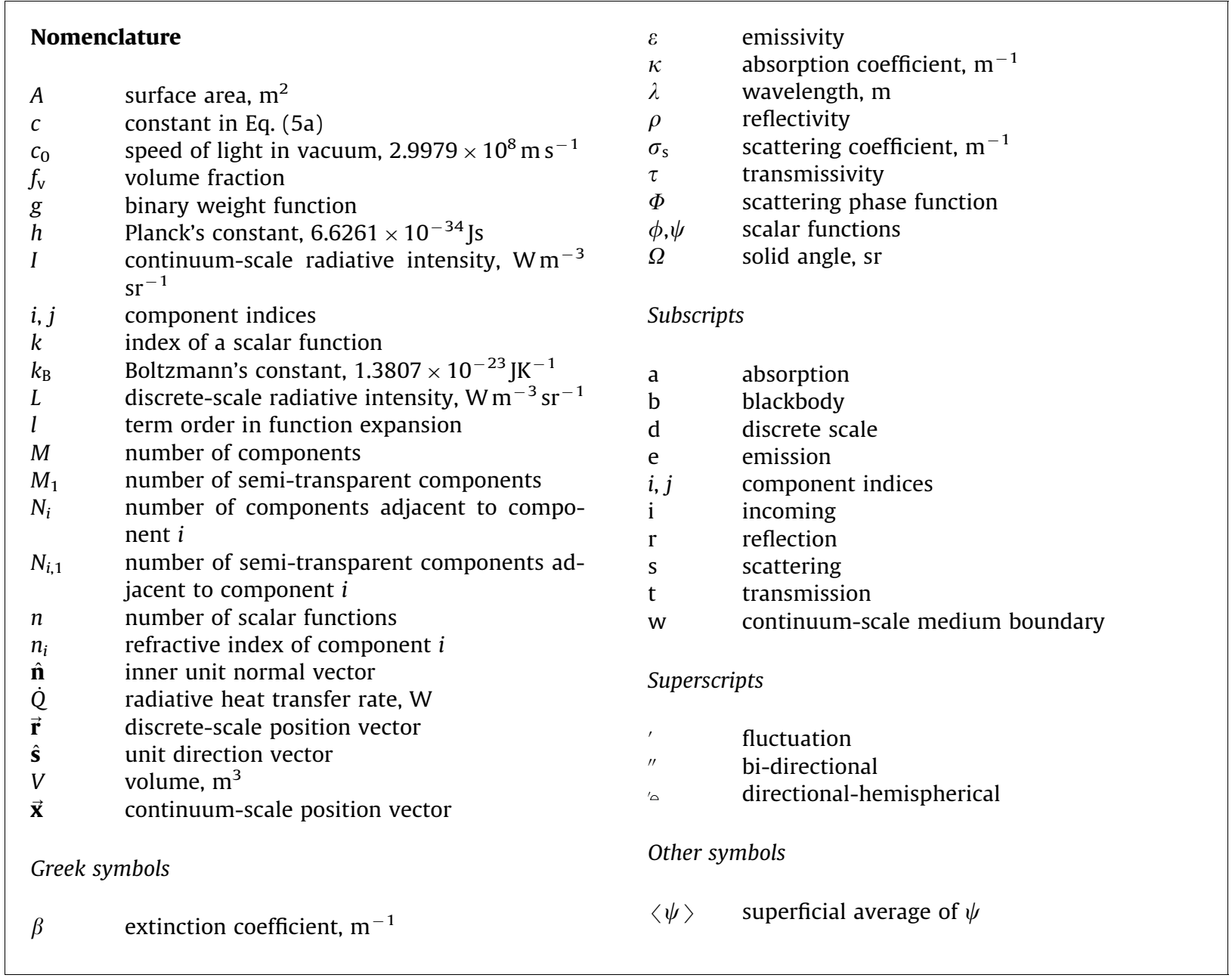

media consisting of an optically-thin component and a semi-transparent component [9-13]. These studies show that the discrete-scale interface and component internal properties, and the exact internal medium geometry (morphology) must be known in order to predict the continuum-scale radiative characteristics. In the studies $[5,7-9,13]$, the exact geometry of porous media with irregular boundaries was obtained by employing X-ray computed tomography. Continuum-scale RTEs were derived for a medium consisting of large opaque and spherical particles suspended in a participating gas phase [14], for a medium consisting of two arbitrary-type and arbitrary-shaped components [15-18], and formulated for a medium consisting of two arbitrary-shaped semitransparent components [9].

In this paper, we generalize the analysis presented in [18] to multi-component media consisting of $M$ arbitrary-type and arbitrary-shaped components in the limit of geometrical optics [19]. A system of continuumscale RTEs and the corresponding boundary conditions are derived by applying volume averaging to discrete-scale RTEs and boundary conditions. A single-RTE approach is formulated for problems, where the overall radiative fluxes transmitted and/or reflected by a multi-component medium are of primary interest. The derivations presented are aimed at providing an analytical basis for development of 'ray optics' based numerical techniques such as the Monte Carlo ray tracing that can be used to radiatively characterize media using their structure and composition. More general derivations of the RTE for media containing arbitrarily shaped and sized components, and accounting for polarization effects, can be found in [20-22].

\section{Basic definitions}

Consider the averaging volume $V$ of a multi-component medium consisting of $M$ components. Each component $i=1, \ldots, M$ is characterized by its partial volume $V_{i}$ and the corresponding volume fraction $f_{\mathrm{v}, i}$. A scalar quantity $\psi_{i}$ for component $i$ can be expressed as

$\psi_{i}=\left\langle\psi_{i}\right\rangle+\psi_{i}^{\prime}$,

where the superficial average, $\left\langle\psi_{i}\right\rangle$, is defined as

$$
\left\langle\psi_{i}\right\rangle \stackrel{\text { def }}{=} \frac{1}{V} \int_{V} \psi_{i} \mathrm{~d} V, \quad V=\sum_{i=1}^{M} V_{i},
$$


Assume that the scalar function $\psi_{i}$ can be expressed as a product of $n$ scalar functions $\psi_{i, k}, k=1, \ldots, n$,

$\psi_{i}=\prod_{k=1}^{n} \psi_{i, k}$

and each of the scalar functions $\psi_{i, k}$ vanishes outside the component $i$, i.e. it can be written in the form

$\psi_{i, k}(\overrightarrow{\mathbf{r}})=\phi_{i, k}(\overrightarrow{\mathbf{r}}) g_{i}(\overrightarrow{\mathbf{r}}), \quad k=1, \ldots, n$.

$\phi_{i, k}(\overrightarrow{\mathbf{r}})$ are scalar functions and $g_{i}(\overrightarrow{\mathbf{r}})$ is a normalized binary weight function,

$g_{i}(\overrightarrow{\mathbf{r}})= \begin{cases}c, & \overrightarrow{\mathbf{r}} \in V_{i}, \\ 0, & \overrightarrow{\mathbf{r}} \in V-V_{i},\end{cases}$

$f_{\mathrm{v}, i}=\frac{1}{V} \int_{V} g_{i}(\overrightarrow{\mathbf{r}}) \mathrm{d} V$,

from which it immediately follows that $c=1$. The first $n-1$ functions $\phi_{i, k}(\overrightarrow{\mathbf{r}})$ are required to be smooth in the averaging volume $V$. Let $\overrightarrow{\mathbf{r}}_{k}, k=1, \ldots, n-1$, be points inside the averaging volume $V$ such that

$\phi_{i, k}\left(\overrightarrow{\mathbf{r}}_{k}\right)=\left\langle\phi_{i, k}\right\rangle, \quad k=1, \ldots, n-1$.

Functions $\phi_{i, k}, k=1, \ldots, n-1$, are developed by using the Taylor expansion,

$\phi_{i, k}(\overrightarrow{\mathbf{r}})=\left\langle\phi_{i, k}\right\rangle+\left.\left|\overrightarrow{\mathbf{r}}-\overrightarrow{\mathbf{r}}_{k}\right| \frac{\mathrm{d} \phi_{i, k}}{\mathrm{~d}\left|\overrightarrow{\mathbf{r}}-\overrightarrow{\mathbf{r}}_{k}\right|}\right|_{\overrightarrow{\mathbf{r}}_{k}}+O\left(\left|\overrightarrow{\mathbf{r}}-\overrightarrow{\mathbf{r}}_{k}\right|^{2}\right), \quad k=1, \ldots, n-1$,

where $O$ denotes the 'big $O$ ' Landau order symbol. For functions $\phi_{i, k}, k=1, \ldots, n-1$, varying weakly in the averaging volume $V$,

$O\left(\left|\overrightarrow{\mathbf{r}}-\overrightarrow{\mathbf{r}}_{k}\right|^{l}\right) \approx 0, \quad l \geq 1, \quad k=1, \ldots, n-1$.

Therefore, the fluctuations in the first $n-1$ functions $\phi_{i, k}$ vanish,

$\phi_{i, 1}^{\prime}(\overrightarrow{\mathbf{r}}) \approx 0$,

$\phi_{i, n-1}^{\prime}(\overrightarrow{\mathbf{r}}) \approx 0$.

Consequently, the superficial average of the scalar function $\psi_{i}$ can be written as

$\left\langle\psi_{i}\right\rangle=\left\langle\prod_{k=1}^{n} \psi_{i, k}\right\rangle \approx\left\langle\psi_{i, n}\right\rangle c^{n-1} \prod_{k=1}^{n-1} \phi_{i, k}$.

The relation between the superficial average of the discrete-scale gradient of $\psi_{i}$ and the continuum-scale gradient of $\left\langle\psi_{i}\right\rangle$ is given by the spatial averaging theorem (SAT) $[23,24]$ :

$$
\left\langle\nabla_{\overrightarrow{\mathbf{r}}} \psi_{i}\right\rangle=\nabla_{\overrightarrow{\mathbf{x}}}\left\langle\psi_{i}\right\rangle-\frac{1}{V} \sum_{j=1}^{N_{i}} \int_{A_{i j}} \psi_{i} \hat{\mathbf{n}}_{j i} \mathrm{~d} A,
$$

where $\overrightarrow{\mathbf{r}}$ and $\overrightarrow{\mathbf{x}}$ are the discrete-scale and continuum-scale position vectors, respectively [23]. $A_{i j}$ is the interface surface area between the component $i$ and the component $j$ adjacent to $i$ in the averaging volume $V . N_{i}$ is the total number of components adjacent to the component $i$. $\hat{\mathbf{n}}_{j i}$ is the inner unit normal vector at the point $\overrightarrow{\mathbf{r}}_{i j}$ of the interface $A_{i j}$, i.e. $\hat{\mathbf{n}}_{j i}$ points into the component $i$.

Finally, the averaging volume $V$ is assumed to be (i) sufficiently large to include all typical morphological structures of the multi-component medium and (ii) sufficiently small as compared to the overall size of the multi-component medium so that $\langle\psi\rangle$ and $\left\langle\nabla_{\overrightarrow{\mathbf{r}}} \psi_{i}\right\rangle$ can be assumed to be continuous scalar and vector fields, respectively.

\section{Continuum-scale equations of radiative transfer}

Consider a multi-component medium consisting of $i=1, \ldots, M_{1}$ and $i=M_{1}+1, \ldots, M$ semi-transparent and opaque components, respectively, each of an arbitrary shape. Each component $i$ is adjacent to $j=1, \ldots, N_{i, 1}$ and $j=N_{i, 1}+1, \ldots, N_{i}$ semi-transparent and opaque components, respectively (see Fig. 1). The analysis presented is subject to the following assumptions: (i) all components are isotropic; (ii) all components are non-polarizing and the state of polarization can be neglected; (iii) all components are at local thermodynamic equilibrium; (iv) characteristic dimensions of all components are much larger than the radiation wavelengths of interest so that laws of geometrical optics are valid in each component; (v) diffraction effects are negligible; (vi) dependentscattering effects are negligible; (vii) all components are at rest as compared to the speed of light; (viii) radiative transfer in each component is quasi-steady. Each component $i$ is characterized by the set of the discrete-scale optical and radiative properties: the effective refractive index $n_{i}=$ const, the absorption and scattering coefficients, $\kappa_{\mathrm{d}, i}$ and $\sigma_{\mathrm{s}, \mathrm{d}, i}$, respectively, and the scattering phase function $\Phi_{\mathrm{d}, i} \cdot \sigma_{\mathrm{s}, \mathrm{d}, i}$ and $\Phi_{\mathrm{d}, i}$ are introduced to account for possible internal microscopic inhomogeneities of the components, and are to be determined by employing theories appropriate for

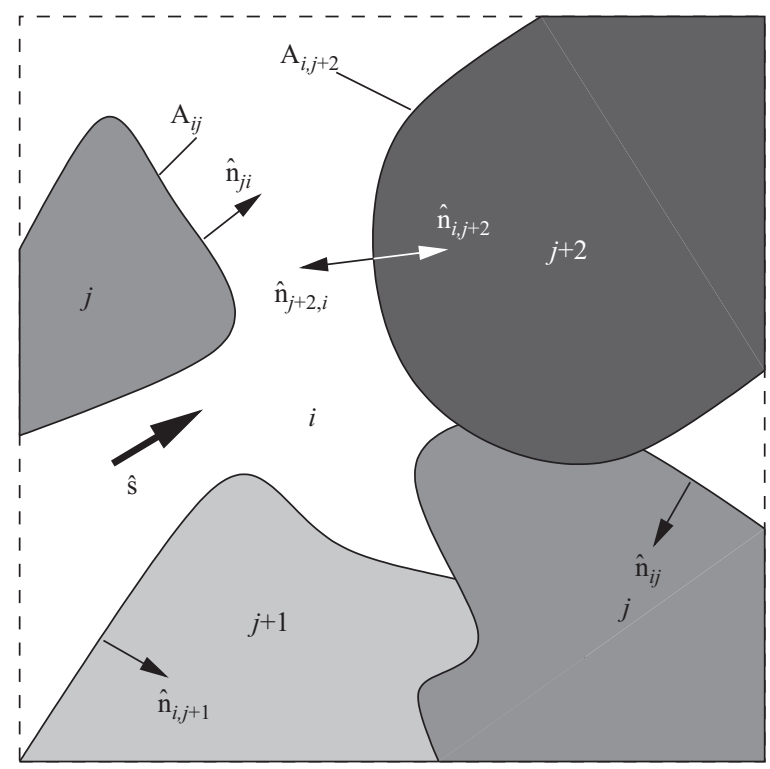

Fig. 1. Multi-component medium with component designation. 
characteristic length scales of the inhomogeneities. Furthermore, each component $i$ is characterized by its temperature $T_{i}$, i.e. the components are allowed to be at thermal non-equilibrium with respect to each other.

The quasi-steady discrete-scale intensity in each component $i$ can be determined by solving the corresponding quasi-steady discrete-scale RTEs [25-27]:

$$
\begin{aligned}
\hat{\mathbf{s}} \cdot \nabla_{\overrightarrow{\mathbf{r}}} L_{i}(\overrightarrow{\mathbf{r}}, \hat{\mathbf{s}})= & -\beta_{\mathrm{d}, i}(\overrightarrow{\mathbf{r}}) L_{i}(\overrightarrow{\mathbf{r}}, \hat{\mathbf{s}})+\kappa_{\mathrm{d}, i}(\overrightarrow{\mathbf{r}}) L_{\mathrm{b}, i}(\overrightarrow{\mathbf{r}}) \\
& +\frac{\sigma_{\mathrm{s}, \mathrm{d}, i}(\overrightarrow{\mathbf{r}})}{4 \pi} \int_{\Omega_{\mathrm{i}}=0}^{4 \pi} L_{i}\left(\overrightarrow{\mathbf{r}}, \hat{\mathbf{s}}_{\mathrm{i}}\right) \Phi_{\mathrm{d}, i}\left(\overrightarrow{\mathbf{r}}, \hat{\mathbf{s}}_{\mathrm{i}}, \hat{\mathbf{s}}\right) \mathrm{d} \Omega_{\mathrm{i}},
\end{aligned}
$$

$i=1, \ldots, M$,

where the spectral subscript $\lambda$ has been omitted for brevity. $L_{\mathrm{b}, i}$ is the spectral blackbody intensity inside the component $i$. For the constant refractive index $n_{i}$, it is given by

$L_{\mathrm{b}, \mathrm{i}}(\overrightarrow{\mathbf{r}})=\frac{2 h c_{0}^{2}}{n_{i}^{2} \lambda^{5}\left[\exp \left(\frac{h c_{0}}{n_{i} k_{\mathrm{B}} \lambda T_{i}(\overrightarrow{\mathbf{r}})}-1\right)\right]}$

and $\lambda$ is radiation wavelength in the component $i$. While Eq. (12) and, consequently, the following analysis requires the effective refractive index to be constant within a component, further reading on treatment of media with graded refractive index can be found in [28,29]. Eq. (12) is subject to the following boundary condition at $A_{i j, \hat{\mathbf{s}} \cdot \hat{\mathbf{n}}_{j i}>0}$ :

$$
\begin{aligned}
L_{i}\left(\overrightarrow{\mathbf{r}}_{i j}, \hat{\mathbf{s}}\right)= & \int_{\Omega_{\hat{\mathbf{s}}_{\mathrm{i}} \hat{\mathbf{n}}_{j i}>0}} \tau_{j i}{ }^{\prime \prime}\left(\overrightarrow{\mathbf{r}}_{i j}, \hat{\mathbf{s}}_{\mathrm{i}}, \hat{\mathbf{s}}\right) L_{j}\left(\overrightarrow{\mathbf{r}}_{i j}, \hat{\mathbf{s}}_{\mathrm{i}}\right) \hat{\mathbf{s}}_{\mathrm{i}} \cdot \hat{\mathbf{n}}_{j i} \mathrm{~d} \Omega_{\mathrm{i}} \\
& -\int_{\Omega_{\hat{\mathbf{s}}_{\mathrm{i}} \mathbf{n}_{j i}<0}} \rho_{i j}{ }^{\prime \prime}\left(\overrightarrow{\mathbf{r}}_{i j}, \hat{\mathbf{s}}_{\mathrm{i}}, \hat{\mathbf{s}}\right) L_{i}\left(\overrightarrow{\mathbf{r}}_{i j}, \hat{\mathbf{s}}_{\mathrm{i}}\right) \hat{\mathbf{s}}_{i} \cdot \hat{\mathbf{n}}_{j i} \mathrm{~d} \Omega_{\mathrm{i}},
\end{aligned}
$$

$j=1, \ldots, N_{i}$,

where $\overrightarrow{\mathbf{r}}_{i j}$ is a position vector at the interface $A_{i j}$. The bidirectional reflection and transmission functions, $\rho^{\prime \prime}{ }_{i j}$ and $\tau^{\prime \prime}{ }_{i j}$, respectively, must satisfy the condition of radiative intensity conservation for incidence at a reflectingtransmitting boundary:

$$
\begin{aligned}
& \int_{\Omega_{\hat{s} \cdot \hat{n}_{j i}>0}} \rho_{i j}{ }^{\prime \prime}\left(\overrightarrow{\mathbf{r}}_{i j}, \hat{\mathbf{s}}_{\mathrm{i}}, \hat{\mathbf{s}}\right) \hat{\mathbf{s}} \cdot \hat{\mathbf{n}}_{j i} \mathrm{~d} \Omega-\int_{\Omega_{s \cdot \hat{\mathbf{n}}_{j i}<0}} \tau_{i j}{ }^{\prime \prime}\left(\overrightarrow{\mathbf{r}}_{i j}, \hat{\mathbf{s}}_{\mathrm{i}}, \hat{\mathbf{s}}\right) \hat{\mathbf{s}} \cdot \hat{\mathbf{n}}_{j i} \mathrm{~d} \Omega \\
& =\rho_{i j}^{\prime} t\left(\overrightarrow{\mathbf{r}}_{i j}, \hat{\mathbf{s}}_{\mathrm{i}}\right)+\tau_{i j}^{\prime} t\left(\overrightarrow{\mathbf{r}}_{i j}, \hat{\mathbf{s}}_{\mathrm{i}}\right)=1, \quad \hat{\mathbf{s}}_{\mathrm{i}} \cdot \hat{\mathbf{n}}_{j i}<0 .
\end{aligned}
$$

For media with small discontinuities present between the components as compared to the radiation wavelengths of interest and to the characteristic dimensions of the components, $\rho_{i j}{ }^{\prime \prime}$ and $\tau_{i j}{ }^{\prime \prime}$ are to be determined by including micro-scale radiative transfer effects at the interfaces. The intensity $L_{j}$ in the boundary condition (14) can formally be obtained by solving Eq. (12) for all components $j=1, \ldots, N_{i}$. However, for opaque components $j=N_{i, 1}+1, \ldots, N_{i}$, the absorption coefficient approaches infinity, $\kappa_{j} \rightarrow \infty$, and the complete solutions to Eq. (12) for these components are not required. A closer inspection of Eq. (12) shows that the intensity $L_{j}\left(\overrightarrow{\mathbf{r}}_{i j}, \hat{\mathbf{s}}\right)$ results only from local emission within the component $j$ in the vicinity of the interface. This allows us to rewrite Eqs. (12) and (14) as

$$
\begin{aligned}
\hat{\mathbf{s}} \cdot \nabla_{\overrightarrow{\mathbf{r}}} L_{i}(\overrightarrow{\mathbf{r}}, \hat{\mathbf{s}})= & -\beta_{\mathrm{d}, i}(\overrightarrow{\mathbf{r}}) L_{i}(\overrightarrow{\mathbf{r}}, \hat{\mathbf{s}})+\kappa_{\mathrm{d}, i}(\overrightarrow{\mathbf{r}}) L_{\mathrm{b}, i}(\overrightarrow{\mathbf{r}}) \\
& +\frac{\sigma_{\mathrm{s}, \mathrm{d}, i}(\overrightarrow{\mathbf{r}})}{4 \pi} \int_{\Omega_{\mathrm{i}}=0}^{4 \pi} L_{i}\left(\overrightarrow{\mathbf{r}}, \hat{\mathbf{s}}_{\mathrm{i}}\right) \Phi_{\mathrm{d}, i}\left(\overrightarrow{\mathbf{r}}, \hat{\mathbf{s}}_{\mathrm{i}}, \hat{\mathbf{s}}\right) \mathrm{d} \Omega_{\mathrm{i}}, \\
i=1, \ldots, M_{1}, &
\end{aligned}
$$

$$
\begin{aligned}
& L_{i}\left(\overrightarrow{\mathbf{r}}_{i j}, \hat{\mathbf{s}}\right)=\int_{\Omega_{\hat{\mathbf{s}}_{i} \cdot \mathbf{n}_{j i}>0}} \tau_{j i}{ }^{\prime \prime}\left(\overrightarrow{\mathbf{r}}_{i j}, \hat{\mathbf{s}}_{\mathrm{i}}, \hat{\mathbf{s}}\right) L_{j}\left(\overrightarrow{\mathbf{r}}_{i j}, \hat{\mathbf{s}}_{\mathrm{i}}\right) \hat{\mathbf{s}}_{\mathrm{i}} \cdot \hat{\mathbf{n}}_{j i} \mathrm{~d} \Omega_{\mathrm{i}} \\
& \quad-\int_{\Omega_{\hat{\mathbf{s}}_{i} \cdot \hat{\mathbf{n}}_{j i}<0}} \rho_{i j}{ }^{\prime \prime}\left(\overrightarrow{\mathbf{r}}_{i j}, \hat{\mathbf{s}}_{\mathrm{i}}, \hat{\mathbf{s}}\right) L_{i}\left(\overrightarrow{\mathbf{r}}_{i j}, \hat{\mathbf{s}}_{\mathrm{i}}\right) \hat{\mathbf{s}}_{\mathrm{i}} \cdot \hat{\mathbf{n}}_{j i} \mathrm{~d} \Omega_{\mathrm{i}}, \\
& j=1, \ldots, N_{i, 1}, \\
& L_{i}\left(\overrightarrow{\mathbf{r}}_{i j}, \hat{\mathbf{s}}\right)=\varepsilon_{j i}^{\prime}\left(\overrightarrow{\mathbf{r}}_{i j}, \hat{\mathbf{s}}\right) L_{\mathrm{b}, j}\left(\overrightarrow{\mathbf{r}}_{i j}\right)-\int_{\Omega_{\hat{s}_{\mathrm{i}}, \mathbf{n}_{j i}<0}} \rho_{i j}{ }^{\prime \prime}\left(\overrightarrow{\mathbf{r}}_{i j}, \hat{\mathbf{s}}_{\mathrm{i}}, \hat{\mathbf{s}}\right) L_{i}\left(\overrightarrow{\mathbf{r}}_{i j}, \hat{\mathbf{s}}_{\mathrm{i}}\right) \hat{\mathbf{s}}_{i} \cdot \hat{\mathbf{n}}_{j i} \mathrm{~d} \Omega_{\mathrm{i}}, \\
& j=N_{i, 1}+1, \ldots, N_{i},
\end{aligned}
$$

where $\varepsilon_{j i}^{\prime}$ is the directional spectral emissivity of the interface between components $j$ and $i$ defined as

$\varepsilon_{j i}^{\prime}\left(\overrightarrow{\mathbf{r}}_{i j}, \hat{\mathbf{s}}\right) \stackrel{\text { def }}{=} \frac{L_{\mathrm{e}, i\left(\overrightarrow{\mathbf{r}}_{i j}, \hat{\mathbf{s}}\right)}}{L_{\mathrm{b}, j}\left(\overrightarrow{\mathbf{r}}_{i j}\right)}$.

$L_{\mathrm{b}, j}\left(\overrightarrow{\mathbf{r}}_{i j}\right)$ is the blackbody intensity emitted by the component $j$ into the component $i$,

$$
L_{\mathrm{b}, j}\left(\overrightarrow{\mathbf{r}}_{i j}\right)=\frac{2 h c_{0}^{2}}{n_{i}^{2} \lambda^{5}\left[\exp \left(\frac{h c_{0}}{n_{i} k_{\mathrm{B}} \lambda T_{j}\left(\overrightarrow{\mathbf{r}}_{i j}\right)}-1\right)\right]} .
$$

Note that the interface emissivity $\varepsilon_{j i}^{\prime}\left(\overrightarrow{\mathbf{r}}_{i j}, \hat{\mathbf{s}}\right)$ captures the interface transmission from the component $j$ into the component $i$ and the definition (18) follows the definition of the emissivity of opaque surfaces [26].

The condition of radiative intensity conservation at the interface between the semi-transparent component $i$ and the opaque component $j$ can be written analogously to Eq. (15),

$$
\begin{aligned}
& \int_{\Omega_{\hat{s} . \hat{\mathbf{n}}_{j i}>0}} \rho_{i j}{ }^{\prime \prime}\left(\overrightarrow{\mathbf{r}}_{i j}, \hat{\mathbf{s}}_{\mathrm{i}}, \hat{\mathbf{s}}\right) \hat{\mathbf{s}} \cdot \hat{\mathbf{n}}_{j i} \mathrm{~d} \Omega+\alpha_{i j}^{\prime}\left(\overrightarrow{\mathbf{r}}_{i j}, \hat{\mathbf{s}}_{\mathrm{i}}\right)=\rho_{i j}^{\prime} t\left(\overrightarrow{\mathbf{r}}_{i j}, \hat{\mathbf{s}}_{\mathrm{i}}\right)+\alpha_{i j}^{\prime}\left(\overrightarrow{\mathbf{r}}_{i j}, \hat{\mathbf{s}}_{\mathrm{i}}\right)=1, \\
& \hat{\mathbf{s}}_{\mathrm{i}} \cdot \hat{\mathbf{n}}_{j i}<0,
\end{aligned}
$$

where Kirchhoffs law,

$\alpha_{i j}^{\prime}\left(\overrightarrow{\mathbf{r}}, \hat{\mathbf{s}}_{\mathrm{i}}\right)=\varepsilon_{j i}^{\prime}\left(\overrightarrow{\mathbf{r}},-\hat{\mathbf{s}}_{\mathrm{i}}\right)$,

has been applied. The spectral superficial average intensity associated with the component $i$ is defined as

$$
\left\langle L_{i}(\overrightarrow{\mathbf{r}}, \hat{\mathbf{s}})\right\rangle \equiv I_{i}(\overrightarrow{\mathbf{x}}, \hat{\mathbf{s}}) \stackrel{\text { def }}{=} \frac{\mathrm{d} \dot{Q}(\overrightarrow{\mathbf{x}}, \hat{\mathbf{s}})}{\mathrm{d} \lambda \mathrm{d} A \mathrm{~d} \Omega},
$$

where $d \dot{Q}(\overrightarrow{\mathbf{x}}, \hat{\mathbf{s}})$ is the spectral radiative heat transfer rate in the wavelength interval $\mathrm{d} \lambda$, the solid angle $\mathrm{d} \Omega$ around direction $\hat{\mathbf{s}}$, and through the surface area $\mathrm{d} A$ of the multicomponent medium normal to $\hat{\mathbf{s}}$. Applying Eq. (2) to each term of Eq. (16) leads to

$$
\begin{aligned}
\left\langle\hat{\mathbf{s}} \cdot \nabla_{\overrightarrow{\mathbf{r}}} L_{i}(\overrightarrow{\mathbf{r}}, \hat{\mathbf{s}})\right\rangle=- & \left\langle\beta_{\mathrm{d}, i}(\overrightarrow{\mathbf{r}}) L_{i}(\overrightarrow{\mathbf{r}}, \hat{\mathbf{s}})\right\rangle+\left\langle\kappa_{\mathrm{d}, i}(\overrightarrow{\mathbf{r}}) L_{\mathrm{b}, i}(\overrightarrow{\mathbf{r}})\right\rangle \\
& +\frac{1}{4 \pi}\left\langle\sigma_{\mathrm{s}, \mathrm{d}, i}(\overrightarrow{\mathbf{r}}) \int_{\Omega_{\mathrm{i}}=0}^{4 \pi} L_{i}\left(\overrightarrow{\mathbf{r}}, \hat{\mathbf{s}}_{\mathrm{i}}\right) \Phi_{\mathrm{d}, i}\left(\overrightarrow{\mathbf{r}}, \hat{\mathbf{s}}_{\mathrm{i}}, \hat{\mathbf{s}}\right) \mathrm{d} \Omega_{\mathrm{i}}\right\rangle,
\end{aligned}
$$

$i=1, \ldots, M_{1}$.

The left-hand side of Eq. (23) is developed by applying SAT, Eq. (11). The terms on the right-hand side of Eq. (23) are developed by applying Eq. (10), i.e. the variation of the discrete-scale radiative properties is small enough so that the corresponding variation of the discrete scale radiative properties inside the averaging volume $V$ becomes negligible. Interchanging the order of integration with respect to the solid angle $\Omega_{\mathrm{i}}$ and the volume $V$ in the incoming scattering 
term results in

$$
\begin{aligned}
\hat{\mathbf{s}} \cdot & \nabla_{\overrightarrow{\mathbf{x}}} I_{i}(\overrightarrow{\mathbf{x}}, \hat{\mathbf{s}})=-\beta_{\mathrm{d}, i}(\overrightarrow{\mathbf{x}}) I_{i}(\overrightarrow{\mathbf{x}}, \hat{\mathbf{s}})+\kappa_{\mathrm{d}, i}(\overrightarrow{\mathbf{x}}) I_{\mathrm{b}, i}(\overrightarrow{\mathbf{x}}) \\
& +\frac{\sigma_{\mathrm{s}, \mathrm{d}, i}(\overrightarrow{\mathbf{x}})}{4 \pi} \int_{\Omega_{\mathrm{i}}=0}^{4 \pi} I_{i}\left(\overrightarrow{\mathbf{x}}, \hat{\mathbf{s}}_{\mathrm{i}}\right) \Phi_{\mathrm{d}, i}\left(\overrightarrow{\mathbf{x}}, \hat{\mathbf{s}}_{\mathrm{i}}, \hat{\mathbf{s}}\right) \mathrm{d} \Omega_{\mathrm{i}} \\
& +\frac{1}{V} \sum_{j=1}^{N_{i}} \int_{A_{i j}} L_{i}(\overrightarrow{\mathbf{r}}, \hat{\mathbf{s}}) \hat{\mathbf{s}} \cdot \hat{\mathbf{n}}_{j i} \mathrm{~d} A \quad i=1, \ldots, M_{1} .
\end{aligned}
$$

The last term on the right-hand side of Eq. (24) represents the contribution to the spectral superficial average radiative heat transfer rate per unit volume and solid angle around the direction $\hat{\mathbf{s}}$ by radiative intensity in component $i$ launched at $A_{i j, \hat{\mathbf{s}} \cdot \hat{\mathbf{n}}_{j i}>0}$ into the direction $\hat{\mathbf{s}}$ and removed in component $i$ at $A_{i j, \hat{\mathbf{s}} \cdot \hat{\mathbf{n}}_{j i}<0}$ from the direction $\hat{\mathbf{s}}$, respectively. Thus, the surface integral in Eq. (24) is split into two parts,

$\int_{A_{i j}} L_{i}(\overrightarrow{\mathbf{r}}, \hat{\mathbf{s}}) \hat{\mathbf{s}} \cdot \hat{\mathbf{n}}_{j i} \mathrm{~d} A=\int_{A_{j, j} \cdot \hat{\mathbf{n}}_{j i}>0} L_{i}(\overrightarrow{\mathbf{r}}, \hat{\mathbf{s}}) \hat{\mathbf{s}} \cdot \hat{\mathbf{n}}_{j i} \mathrm{~d} A+\int_{A_{i j, \hat{s} \cdot \hat{\mathbf{n}}_{j i}<0}} L_{i}(\overrightarrow{\mathbf{r}}, \hat{\mathbf{s}}) \hat{\mathbf{s}} \cdot \hat{\mathbf{n}}_{j i} \mathrm{~d} A$.

The first term on the right-hand side of Eq. (25) is developed by using the boundary conditions, Eqs. (17a) and (17b). The second term on the right-hand side of Eq. (25) is developed by using Eqs. (15) and (20). Eq. (25) becomes

$$
\begin{aligned}
& \int_{A_{i}} L_{i}(\overrightarrow{\mathbf{r}}, \hat{\mathbf{s}}) \hat{\mathbf{s}} \cdot \hat{\mathbf{n}}_{j i} \mathrm{~d} A=\sum_{j=N_{i, 1}+1}^{N_{i}} \int_{A_{i, j, \hat{\mathbf{s}} . \hat{\mathbf{n}}_{j i}>0}} \varepsilon_{j i}^{\prime}\left(\overrightarrow{\mathbf{r}}_{i j}, \hat{\mathbf{s}}\right) L_{\mathrm{b}, j}\left(\overrightarrow{\mathbf{r}}_{i j}\right) \hat{\mathbf{s}} \cdot \hat{\mathbf{n}}_{j i} \mathrm{~d} A \\
& -\sum_{j=1}^{N_{i}} \int_{A_{i j, \hat{\mathbf{s}} \cdot \hat{\mathbf{n}}_{j i}>0}} \int_{\Omega_{\hat{\mathbf{s i}}} \cdot \hat{\mathbf{n}}_{j i}<0} \rho_{i j}{ }^{\prime \prime}\left(\overrightarrow{\mathbf{r}}, \hat{\mathbf{s}}_{\mathrm{i}}, \hat{\mathbf{s}}\right) L_{i}\left(\overrightarrow{\mathbf{r}}, \hat{\mathbf{s}}_{\mathrm{i}}\right) \hat{\mathbf{s}}_{\mathrm{i}} \cdot \hat{\mathbf{n}}_{j i} \mathrm{~d} \Omega_{\mathrm{i}} \hat{\mathbf{s}} \cdot \hat{\mathbf{n}}_{j i} \mathrm{~d} A \\
& +\sum_{j=1}^{N_{i, 1}} \int_{A_{i j, \hat{s} \cdot \hat{\mathbf{n}}_{j i}>0}} \int_{\Omega_{\hat{s}_{\mathrm{i}}} \cdot \hat{\mathbf{n}}_{j i}>0} \tau_{j i}{ }^{\prime \prime}\left(\overrightarrow{\mathbf{r}}, \hat{\mathbf{s}}_{\mathrm{i}}, \hat{\mathbf{s}}\right) L_{j}\left(\overrightarrow{\mathbf{r}}, \hat{\mathbf{s}}_{\mathrm{i}}\right) \hat{\mathbf{s}}_{\mathrm{i}} \cdot \hat{\mathbf{n}}_{j i} \mathrm{~d} \Omega_{\mathrm{i}} \hat{\mathbf{s}} \cdot \hat{\mathbf{n}}_{j i} \mathrm{~d} A \\
& +\sum_{j=N_{i, 1+1}}^{N_{i}} \int_{A_{i j, \hat{s} \cdot \hat{n}_{j i}<0}} \alpha_{i j}^{\prime}(\overrightarrow{\mathbf{r}}, \hat{\mathbf{s}}) L_{i}(\overrightarrow{\mathbf{r}}, \hat{\mathbf{s}}) \hat{\mathbf{s}} \cdot \hat{\mathbf{n}}_{j i} \mathrm{~d} A \\
& +\sum_{j=1}^{N_{i}} \int_{A_{i j, \hat{s}, \hat{n}_{j i}<0}} \rho_{i j}^{\prime} t(\overrightarrow{\mathbf{r}}, \hat{\mathbf{s}}) L_{i}(\overrightarrow{\mathbf{r}}, \hat{\mathbf{s}}) \hat{\mathbf{s}} \cdot \hat{\mathbf{n}}_{j i} \mathrm{~d} A \\
& +\sum_{j=1}^{N_{i, 1}} \int_{A_{i j, \hat{s} \mathbf{s}, \hat{\mathbf{n}}_{j i}<0}} \tau_{i j}^{\prime} t(\overrightarrow{\mathbf{r}}, \hat{\mathbf{s}}) L_{i}(\overrightarrow{\mathbf{r}}, \hat{\mathbf{s}}) \hat{\mathbf{s}} \cdot \hat{\mathbf{n}}_{j i} \mathrm{~d} A
\end{aligned}
$$

The first three terms on the right-hand side of Eq. (26) quantify the augmentation of the superficial average radiative heat transfer rate per unit solid angle around direction $\hat{\mathbf{s}}$ in the component $i$ resulting from the intensities emitted, reflected, and transmitted into the component $i$, respectively. The last three terms on the right-hand side of Eq. (26) quantify the attenuation of the superficial average radiative heat transfer rate per unit solid angle around direction $\hat{\mathbf{s}}$ by interface absorption, reflection, and transmission, respectively. Thus, recalling the definition of the superficial average intensity, Eq. (22), the following absorption and scattering coefficients, and the scattering phase functions associated with the superficial average intensities $I_{i}$ and $I_{\mathrm{b}, j}$ are postulated:

$$
\begin{gathered}
\kappa_{i j}(\overrightarrow{\mathbf{x}}) \stackrel{\text { def }}{=}-\frac{\int_{A_{i j, \hat{s} \cdot \hat{n}_{j i}<0}} \alpha_{i j}^{\prime}\left(\overrightarrow{\mathbf{r}}_{i j}, \hat{\mathbf{s}}\right) L_{i}(\overrightarrow{\mathbf{r}}, \hat{\mathbf{s}}) \hat{\mathbf{s}} \cdot \hat{\mathbf{n}}_{j i} \mathrm{~d} A}{I_{i}(\overrightarrow{\mathbf{x}}, \hat{\mathbf{s}}) V}, \\
\kappa_{j i}(\overrightarrow{\mathbf{x}}) \stackrel{\operatorname{def}}{=} \frac{\int_{A_{i j, \hat{s} \cdot \hat{\mathbf{n}} \hat{n}_{i i}>0}} \varepsilon_{j i}^{\prime}\left(\overrightarrow{\mathbf{r}}_{i j}, \hat{\mathbf{s}}\right) L_{\mathrm{b}, j}(\overrightarrow{\mathbf{r}}, \hat{\mathbf{s}}) \hat{\mathbf{s}} \cdot \hat{\mathbf{n}}_{j i} \mathrm{~d} A}{I_{\mathrm{b}, j}(\overrightarrow{\mathbf{x}}, \hat{\mathbf{s}}) V},
\end{gathered}
$$

$$
\begin{aligned}
& \sigma_{\mathrm{s}, \mathrm{r}, i j}(\overrightarrow{\mathbf{x}}) \stackrel{\text { def }}{=}-\frac{\int_{A_{i j, \hat{\mathbf{s}} \cdot \hat{n}_{j i}<0} \rho_{i j}^{\prime} t(\overrightarrow{\mathbf{r}}, \hat{\mathbf{s}}) L_{i}(\overrightarrow{\mathbf{r}}, \hat{\mathbf{s}}) \hat{\mathbf{s}} \cdot \hat{\mathbf{n}}_{j i} \mathrm{~d} A}}{I_{i}(\overrightarrow{\mathbf{x}}, \hat{\mathbf{s}}) V}, \\
& \sigma_{\mathrm{s}, \mathrm{t}, i j}(\overrightarrow{\mathbf{x}}) \stackrel{\text { def }}{=}-\frac{\int_{A_{i j, \hat{s} \cdot \hat{\mathbf{n}}_{j i}<0}} \tau_{i j}^{\prime \circ} t(\overrightarrow{\mathbf{r}}, \hat{\mathbf{s}}) L_{i}(\overrightarrow{\mathbf{r}}, \hat{\mathbf{s}}) \hat{\mathbf{s}} \cdot \hat{\mathbf{n}}_{j i} \mathrm{~d} A}{I_{i}(\overrightarrow{\mathbf{x}}, \hat{\mathbf{s}}) V}, \\
& \Phi_{\mathrm{r}, i j}\left(\overrightarrow{\mathbf{x}}, \hat{\mathbf{s}}_{\mathrm{i}}, \hat{\mathbf{s}}\right) \stackrel{\text { def }}{=}-\frac{\int_{A_{i j, \hat{\mathbf{s}} \cdot \hat{\mathbf{n}}_{j i}>0}} \rho_{i j}{ }^{\prime \prime}\left(\overrightarrow{\mathbf{r}}, \hat{\mathbf{s}}_{\mathrm{i}}, \hat{\mathbf{s}}\right) L_{i}\left(\overrightarrow{\mathbf{r}}, \hat{\mathbf{s}}_{\mathrm{i}}\right) \hat{\mathbf{s}}_{\mathrm{i}} \cdot \hat{\mathbf{n}}_{j i} \hat{\mathbf{s}} \cdot \hat{\mathbf{n}}_{j i} \mathrm{~d} A}{(4 \pi)^{-1} \sigma_{\mathrm{s}, \mathrm{r}, i j} I_{i}(\overrightarrow{\mathbf{x}}, \hat{\mathbf{s}}) V}, \\
& \hat{\mathbf{s}}_{\mathrm{i}} \cdot \hat{\mathbf{n}}_{j i}<0, \\
& \Phi_{\mathrm{t}, j i}\left(\overrightarrow{\mathbf{x}}, \hat{\mathbf{s}}_{\mathrm{i}}, \hat{\mathbf{s}}\right) \stackrel{\text { def }}{=} \frac{\int_{A_{i j,}, \hat{\mathbf{s}}_{\mathrm{i} i}>0} \tau_{j i}{ }^{\prime \prime}\left(\overrightarrow{\mathbf{r}}, \hat{\mathbf{s}}_{\mathrm{i}}, \hat{\mathbf{s}}\right) L_{j}\left(\overrightarrow{\mathbf{r}}, \hat{\mathbf{s}}_{\mathrm{i}}\right) \hat{\mathbf{s}}_{\mathrm{i}} \cdot \hat{\mathbf{n}}_{j i} \hat{\mathbf{s}} \cdot \hat{\mathbf{n}}_{j i} \mathrm{~d} A}{(4 \pi)^{-1} \sigma_{\mathrm{s}, \mathrm{t}, j i} I_{j}(\overrightarrow{\mathbf{x}}, \hat{\mathbf{s}}) V}, \\
& \hat{\mathbf{s}}_{\mathrm{i}} \cdot \hat{\mathbf{n}}_{j i}>0 \text {. }
\end{aligned}
$$

Note that the blackbody intensity $I_{\mathrm{b}, j}$ appearing in Eq. (28) is obtained by applying Eq. (2) to a discrete-scale blackbody intensity that would fill the component $i$ as a result of emission from a black interface $A_{i j, \hat{\mathbf{s}} \cdot \hat{\mathbf{n}}_{j i}>0}$ into the component $i, \varepsilon_{j i}^{\prime}=1$. Substituting Eqs. (25)-(32) into Eq. (24) and omitting for brevity the position vector notation in the radiative properties, results in:

$$
\begin{aligned}
\hat{\mathbf{s}} \cdot \nabla_{\overrightarrow{\mathbf{x}}} I_{i}(\overrightarrow{\mathbf{x}}, \hat{\mathbf{s}})= & -\left[\beta_{\mathrm{d}, i}+\sum_{j=N_{i, 1}+1}^{N_{i}} \kappa_{i j}+\sum_{j=1}^{N_{\mathrm{i}, 1}} \sigma_{\mathrm{s}, \mathrm{t}, i j}+\sum_{j=1}^{N_{i}} \sigma_{\mathrm{s}, \mathrm{r}, i j}\right] I_{i}(\overrightarrow{\mathbf{x}}, \hat{\mathbf{s}}) \\
& +\kappa_{\mathrm{d}, i} I_{\mathrm{b}, i}(\overrightarrow{\mathbf{x}}, \hat{\mathbf{s}})+\sum_{j=N_{i, 1}+1}^{N_{i}} \kappa_{j i} I_{\mathrm{b}, j}(\overrightarrow{\mathbf{x}}, \hat{\mathbf{s}}) \\
& +\frac{\sigma_{\mathrm{s}, \mathrm{d}, i}}{4 \pi} \int_{\Omega_{\mathrm{i}}=0}^{4 \pi} I_{i}\left(\overrightarrow{\mathbf{x}}, \hat{\mathbf{s}}_{\mathrm{i}}\right) \Phi_{\mathrm{d}, i}\left(\hat{\mathbf{s}}_{\mathrm{i}}, \hat{\mathbf{s}}\right) \mathrm{d} \Omega_{\mathrm{i}} \\
& +\sum_{j=1}^{N_{i, 1}} \frac{\sigma_{\mathrm{s}, \mathrm{t}, j i}}{4 \pi} \int_{\Omega_{\mathrm{i}}=0}^{4 \pi} I_{j}\left(\overrightarrow{\mathbf{x}}, \hat{\mathbf{s}}_{\mathrm{i}}\right) \Phi_{\mathrm{t}, j i}\left(\hat{\mathbf{s}}_{\mathrm{i}}, \hat{\mathbf{s}}\right) \mathrm{d} \Omega_{\mathrm{i}} \\
& +\sum_{j=1}^{N_{i}} \frac{\sigma_{\mathrm{s}, \mathrm{r}, i j}}{4 \pi} \int_{\Omega_{\mathrm{i}}=0}^{4 \pi} I_{i}\left(\overrightarrow{\mathbf{x}}, \hat{\mathbf{s}}_{\mathrm{i}}\right) \Phi_{\mathrm{r}, i j}\left(\hat{\mathbf{s}}_{\mathrm{i}}, \hat{\mathbf{s}}\right) \mathrm{d} \Omega_{\mathrm{i}},
\end{aligned}
$$

The set of Eqs. (33) presents generalization of Eq. (1) in [9], Eqs. (8) and (9) in [15], and Eqs. (18) and (27) in [18], for a multi-component medium consisting of any number of semi-transparent and opaque components. To our best knowledge, it is the most complete formulation of continuum radiative transfer in multi-component media with the individual components in the range of geometrical optics.

Eqs. (27)-(32) provide the mathematical basis for development of numerical techniques for determination of continuum-scale radiative properties utilizing the exact geometry of multi-component media. They require the knowledge of the complete actual and blackbody discretescale radiative intensity fields in each component obtained for a selected model problem. The general analytical proof that the resulting continuum-scale radiative properties for a given medium are independent of radiative intensities (boundary conditions applied in the model problem), and depend only on the discretescale radiative properties and morphology, is beyond the scope of the present study. Once the continuum-scale radiative properties are known for a given medium, 
Eqs. (33) can be solved for prescribed boundary conditions by using standard RTE solution techniques. Although the discrete-scale radiative intensities are not longer needed to solve the continuum-scale RTEs, they are still required to formulate the continuum-scale boundary conditions as described in the next section.

\section{Continuum-scale boundary conditions}

Eqs. (33) are subject to boundary conditions at the wall-medium interface at $\hat{\mathbf{s}} \cdot \hat{\mathbf{n}}_{\mathrm{w}}>0$, where $\hat{\mathbf{n}}_{\mathrm{w}}$ is a unit normal vector pointing from the wall into the medium. The wall is assumed to consist of only a single component that can be either semi-transparent or opaque. The discrete-scale boundary conditions at the boundary of the multi-component medium are formulated analogously to the boundary conditions (17a) and (17b). They read for the semi-transparent and opaque walls, respectively:

$$
\begin{aligned}
L_{i}\left(\overrightarrow{\mathbf{r}}_{i \mathrm{w}}, \hat{\mathbf{s}}\right)= & \int_{\Omega_{\hat{\mathbf{s}}_{\mathrm{i}} \cdot \hat{\mathbf{n}}_{\mathrm{w} i}>0}} \tau_{\mathrm{w} i}{ }^{\prime \prime}\left(\overrightarrow{\mathbf{r}}_{i \mathrm{w}}, \hat{\mathbf{s}}_{\mathrm{i}}, \hat{\mathbf{s}}\right) L_{\mathrm{w}}\left(\overrightarrow{\mathbf{r}}_{i \mathrm{w}}, \hat{\mathbf{s}}_{\mathrm{i}}\right) \hat{\mathbf{s}}_{\mathrm{i}} \cdot \hat{\mathbf{n}}_{\mathrm{w} i} \mathrm{~d} \Omega_{\mathrm{i}} \\
& -\int_{\Omega_{\hat{\mathbf{s}}_{\mathrm{i}} \cdot \hat{\mathbf{n}}_{\mathrm{w} i}<0}} \rho_{i \mathrm{w}}{ }^{\prime \prime}\left(\overrightarrow{\mathbf{r}}_{i \mathrm{~W}}, \hat{\mathbf{s}}_{\mathrm{i}}, \hat{\mathbf{s}}\right) L_{i}\left(\overrightarrow{\mathbf{r}}_{i \mathrm{w}}, \hat{\mathbf{s}}_{\mathrm{i}}\right) \hat{\mathbf{s}}_{\mathrm{i}} \cdot \hat{\mathbf{n}}_{\mathrm{w} i} \mathrm{~d} \Omega_{\mathrm{i}},
\end{aligned}
$$

$$
\begin{aligned}
L_{i}\left(\overrightarrow{\mathbf{r}}_{i \mathrm{~W}}, \hat{\mathbf{s}}\right)= & \varepsilon_{\mathrm{wi} i}^{\prime}\left(\overrightarrow{\mathbf{r}}_{i \mathrm{~W}}, \hat{\mathbf{s}}\right) L_{\mathrm{b}, \mathrm{w}}\left(\overrightarrow{\mathbf{r}}_{i \mathrm{~W}}\right) \\
& -\int_{\Omega_{\hat{\mathrm{s}}_{\mathrm{i}} \cdot \hat{\mathbf{n}}_{\mathrm{w} i}<0}} \rho_{i \mathrm{w}}{ }^{\prime \prime}\left(\overrightarrow{\mathbf{r}}_{i \mathrm{~W}}, \hat{\mathbf{s}}_{\mathrm{i}}, \hat{\mathbf{s}}\right) L_{i}\left(\overrightarrow{\mathbf{r}}_{i \mathrm{w}}, \hat{\mathbf{s}}_{\mathrm{i}}\right) \hat{\mathbf{s}}_{\mathrm{i}} \cdot \hat{\mathbf{n}}_{\mathrm{w} i} \mathrm{~d} \Omega_{\mathrm{i}},
\end{aligned}
$$

The variation of the discrete-scale radiative properties and the curvature of the wall-medium interface are assumed to be negligible over the interface area associated with the averaging volume $V$ adjacent to said boundary, $A_{i w, \hat{\mathbf{s}} \cdot \hat{\mathbf{n}}_{w i}>0}$. The continuum-scale boundary conditions are obtained by surface averaging of the boundary intensity $L_{i}\left(\overrightarrow{\mathbf{r}}_{i \mathrm{~W}}, \hat{\mathbf{s}}\right)$,

$I_{i}\left(\overrightarrow{\mathbf{x}}_{\mathrm{w}}, \hat{\mathbf{s}}\right) \stackrel{\text { def }}{=} \frac{\int_{A_{i \mathrm{w}, \hat{\mathbf{s}} \cdot \hat{\mathbf{n}}_{\mathrm{wi}}>0}} L_{i}\left(\overrightarrow{\mathbf{r}}_{i \mathrm{w}}, \hat{\mathbf{s}}\right) \mathrm{d} A}{\int_{A_{\mathrm{w}}, \hat{\mathbf{s}} \cdot \hat{\mathbf{n}}_{\mathrm{w}}>0} \mathrm{~d} A}$,

where $A_{\mathrm{w}}$ is the portion of the wall-medium interface inside the averaging volume $V$ adjacent to the wall. Applying Eq. (35) to Eqs. (34a) and (34b), and interchanging the order of integration with respect to $\Omega_{\mathrm{i}}$ and $A$ on the right-hand side of the resulting equation leads to

$$
\begin{gathered}
I_{i}\left(\overrightarrow{\mathbf{x}}_{\mathrm{w}}, \hat{\mathbf{s}}\right)=\int_{\Omega_{\hat{\mathrm{s}}_{\mathrm{i}} \hat{\mathbf{n}}_{\mathrm{wi}}>0}} \tau_{\mathrm{w} i}{ }^{\prime \prime}\left(\overrightarrow{\mathbf{x}}_{\mathrm{w}}, \hat{\mathbf{s}}_{\mathrm{i}}, \hat{\mathbf{s}}\right) I_{\mathrm{w} i}\left(\overrightarrow{\mathbf{x}}_{\mathrm{w}}, \hat{\mathbf{s}}_{\mathrm{i}}\right) \hat{\mathbf{s}}_{\mathrm{i}} \cdot \hat{\mathbf{n}}_{\mathrm{w} i} \mathrm{~d} \Omega_{\mathrm{i}} \\
-\int_{\Omega_{\hat{\mathrm{s}}_{\mathrm{i}}, \hat{\mathbf{n}}_{\mathrm{w} i}<0}} \rho_{i \mathrm{w}}{ }^{\prime \prime}\left(\overrightarrow{\mathbf{x}}_{\mathrm{w}}, \hat{\mathbf{s}}_{\mathrm{i}}, \hat{\mathbf{s}}\right) I_{i}\left(\overrightarrow{\mathbf{x}}_{\mathrm{w}}, \hat{\mathbf{s}}_{\mathrm{i}}\right) \hat{\mathbf{s}}_{\mathrm{i}} \cdot \hat{\mathbf{n}}_{\mathrm{w} i} \mathrm{~d} \Omega_{\mathrm{i}}, \\
I_{i}\left(\overrightarrow{\mathbf{x}}_{\mathrm{w}}, \hat{\mathbf{s}}\right)=\varepsilon_{\mathrm{w} i}^{\prime}\left(\overrightarrow{\mathbf{x}}_{\mathrm{w}}, \hat{\mathbf{s}}\right) I_{\mathrm{b}, \mathrm{w} i}\left(\overrightarrow{\mathbf{x}}_{\mathrm{w}}, \hat{\mathbf{s}}\right) \\
-\int_{\Omega_{\hat{\mathrm{s}}_{\mathrm{i}} \hat{\mathbf{n}}_{\mathrm{w} i}<0}} \rho_{i \mathrm{w}}{ }^{\prime \prime}\left(\overrightarrow{\mathbf{x}}_{\mathrm{w}}, \hat{\mathbf{s}}_{\mathrm{i}}, \hat{\mathbf{s}}\right) I_{i}\left(\overrightarrow{\mathbf{x}}_{\mathrm{w}}, \hat{\mathbf{s}}_{\mathrm{i}}\right) \hat{\mathbf{s}}_{\mathrm{i}} \cdot \hat{\mathbf{n}}_{\mathrm{w} i} \mathrm{~d} \Omega_{\mathrm{i}} .
\end{gathered}
$$

\section{Single radiative transfer equation approach}

A single radiative transfer equation is typically employed to model radiative transfer in disperse systems. A single intensity through the host phase is sought in this case and the radiative properties of the disperse phase are usually determined by assuming its uniform illumination and low volume fraction [25-27]. For multi-component media with considerable volume fractions of the components in the limit of geometrical optics, a complete solution to the set of Eqs. (33) is generally required as shown in the previous section. Using the following definitions:

$$
\begin{aligned}
& I \stackrel{\text { def }}{=} \sum_{i=1}^{M_{1}} I_{i} \\
& \beta I \stackrel{\text { def }}{=} \sum_{i=1}^{M_{1}}\left(\beta_{\mathrm{d}, i}+\sum_{j=N_{i, 1}+1}^{N_{i}} \kappa_{i j}+\sum_{j=1}^{N_{i, 1}} \sigma_{\mathrm{s}, \mathrm{t}, i j}+\sum_{j=1}^{N_{i}} \sigma_{\mathrm{s}, \mathrm{r}, i j}\right) I_{i}, \\
& \kappa I_{\mathrm{b}} \stackrel{\text { def }}{=} \sum_{i=1}^{M_{1}}\left(\kappa_{\mathrm{d}, i} I_{\mathrm{b}, i}+\sum_{j=N_{i, 1}+1}^{N_{i}} \kappa_{j i} I_{\mathrm{b}, j}\right), \\
& \sigma_{\mathrm{s}} \Phi I \stackrel{\text { deff }}{=} \sum_{i=1}^{M_{1}}\left[\left(\sigma_{\mathrm{s}, d, i} \Phi_{\mathrm{d}, i}+\sum_{j=1}^{N_{i}} \sigma_{\mathrm{s}, r, i j} \Phi_{\mathrm{r}, i j}\right) I_{i}+\sum_{j=1}^{N_{i, 1}} \sigma_{\mathrm{s}, t, j i} \Phi_{\mathrm{t}, j i} I_{j}\right]
\end{aligned}
$$

the set of Eqs. (33) can formally be reduced to a single RTE for a single effective multi-component intensity,

$$
\begin{aligned}
\hat{\mathbf{s}} \cdot & \nabla_{\overrightarrow{\mathbf{x}}} I(\overrightarrow{\mathbf{x}}, \hat{\mathbf{s}})=-\beta(\overrightarrow{\mathbf{x}}) I(\overrightarrow{\mathbf{x}}, \hat{\mathbf{s}})+\kappa(\overrightarrow{\mathbf{x}}) I_{\mathrm{b}}(\overrightarrow{\mathbf{x}}) \\
& +\frac{\sigma_{\mathrm{s}}(\overrightarrow{\mathbf{x}})}{4 \pi} \int_{\Omega_{\mathrm{i}}=0}^{4 \pi} I\left(\overrightarrow{\mathbf{x}}, \hat{\mathbf{s}}_{\mathrm{i}}\right) \Phi\left(\overrightarrow{\mathbf{x}}, \hat{\mathbf{s}}_{\mathrm{i}}, \hat{\mathbf{s}}\right) \mathrm{d} \Omega_{\mathrm{i}} .
\end{aligned}
$$

The effective radiative properties, as defined by Eqs. (37)-(40) depend in this case on the average intensities associated with the individual components, and they cannot in general be separated from the single effective intensity, i.e. the effective radiative properties cannot be viewed as medium physical properties. Moreover, since only a single intensity is used, Eq. (41) cannot be used to model multi-component media at thermal non-equilibrium between the components, where the divergence of radiative flux is needed for individual components.

\section{Summary and conclusions}

In this paper, we have formulated a mathematical model for radiative transfer and characterization of multi-component participating media with the individual components in the limit of geometrical optics. This work has been done in response to a growing number of studies on the development of numerical techniques for radiative transfer modeling and characterization of complex media consisting of components in the limit of geometrical optics. The set of governing continuum-scale equations of radiative transfer, and the corresponding relations for radiative properties and boundary conditions were derived by using the discrete-scale equations of radiative transfer, radiative properties, and boundary conditions by applying the spatial averaging theorem. The formulation presented unifies the two approaches of [18] derived separately for media consisting either of two 
semi-transparent components or of one semi-transparent component and one opaque component.

The relations presented provide a complete mathematical basis for developing new methods for radiative characterization of multi-component media. They are particularly suited for Monte Carlo ray tracing techniques that utilize exact geometry of complex media either generated artificially or obtained experimentally for real media, e.g. by employing tomography techniques. Discrete and continuum modeling approaches pertaining to the analytical formulation presented in this paper have recently been examined for real two-phase media with complex geometries such as reticulate porous ceramics and packed beds of semi-transparent calcium carbonate particles [30].

The analytical derivations show that determination of continuum-scale radiative properties for a particular medium requires in general the knowledge of complete discrete-scale radiative intensity fields in all components and intensity distributions at the medium boundary. These intensities can be obtained by considering a simple radiative transfer model problem involving the medium of interest. The definitions of radiative properties provided in this study will be used in following studies to verify the hypothesis of their independence of radiative boundary conditions applied in a model problem.

\section{References}

[1] Born M, Wolf E. Principles of optics. 7th ed.. Cambridge: Cambridge University Press; 1999.

[2] Baillis D, Sacadura J-F. Thermal radiation properties of dispersed media: theoretical prediction and experimental characterization. Journal of Quantitative Spectroscopy and Radiative Transfer 2000;67:327-63.

[3] Viskanta R, Mengüç MP. Radiative transfer in dispersed media. Applied Mechanics Review 1989;42:241-59.

[4] Tancrez M, Taine J. Direct identification of absorption and scattering coefficients and phase function of a porous medium by a Monte Carlo technique. International Journal of Heat and Mass Transfer 2004;47:373-83.

[5] Petrasch J, Wyss P, Steinfeld A. Tomography-based Monte Carlo determination of radiative properties of reticulate porous ceramics. Journal of Quantitative Spectroscopy and Radiative Transfer 2007; 105:180-97.

[6] Coquard R, Baillis D. Radiative characteristics of opaque spherical particle beds: a new method of prediction. Journal of Thermophysics and Heat Transfer 2004;18:178-86.

[7] Haussener S, Coray P, Lipiński W, Wyss P, Steinfeld A. Tomographybased heat and mass transfer characterization of reticulate porous ceramics for high-temperature processing. Journal of Heat Transfer 2010;132:023305

[8] Haussener S, Lipiński W, Wyss P, Steinfeld A. Tomography-based analysis of radiative transfer in reacting packed beds undergoing a solid-gas thermochemical transformation. Journal of Heat Transfer 2010;132:061201.

[9] Zeghondy B, Iacona E, Taine J. Determination of the anisotropic radiative properties of a porous material by radiative distribution function identification (RDFI). International Journal of Heat and Mass Transfer 2006;49:2810-9.
[10] Zeghondy B, Iacona E, Taine J. Experimental and RDFI calculated radiative properties of a mullite foam. International Journal of Heat and Mass Transfer 2006;49:3702-7.

[11] Coquard R, Baillis D. Radiative characteristics of beds of spheres containing an absorbing and scattering medium. Journal of Thermophysics and Heat Transfer 2005;19:226-34.

[12] Coquard R, Baillis D. Radiative characteristics of beds made of large spheres containing an absorbing and scattering medium. International Journal of Thermal Sciences 2005;44:926-32.

[13] Haussener S, Lipiński W, Petrasch J, Wyss P, Steinfeld A. Tomographic characterization of a semitransparent-particle packed bed and determination of its thermal radiative properties. Journal of Heat Transfer 2009;131:072701.

[14] Consalvi JL, Porterie B, Loraud JC. A formal averaging procedure for radiation heat transfer in particulate media. International Journal of Heat and Mass Transfer 2002;45:2755-68.

[15] Gusarov AV. Homogenization of radiation transfer in two-phase media with irregular phase boundaries. Physical Review B 2008;77:144201.

[16] Gusarov AV. A model of averaged radiation transfer in two-phase heterogeneous medium. High Temperature 2009;47:396-411.

[17] Gusarov AV. Model of radiative heat transfer in heterogeneous multiphase media. Physical Review B 2010;81:064202.

[18] Lipiński W, Petrasch J, Haussener S. Application of the spatial averaging theorem to radiative heat transfer in two-phase media. Journal of Quantitative Spectroscopy and Radiative Transfer 2010;111:253-8

[19] Lipiński W, Keene D, Haussener S, Petrasch J. Continuum radiative heat transfer modeling in media consisting of optically distinct components in the limit of geometrical optics. In: Webb B, Lemonnier $\mathrm{D}$, editors. Proceedings of the 6th international symposium on radiative transfer RAD-10, Antalya; 2010.

[20] Mishchenko MI. Vector radiative transfer equation for arbitrarily shaped and arbitrarily oriented particles: a microphysical derivation from statistical electromagnetics. Applied Optics 2002;41:7114-34.

[21] Mishchenko MI. Radiative transfer theory: from Maxwell's equations to practical applications. In: van Tiggelen $B$ Skipetrov S, editors. Wave scattering in complex media: from theory to applications. Dordrecht: Kluwer Academic Publishers; 2003. p. 367-414.

[22] Mishchenko MI. Maxwell's equations, radiative transfer, and coherent backscattering: a general perspective. Journal of Quantitative Spectroscopy and Radiative Transfer 2006;101: 540-55.

[23] Whitaker S. The method of volume averaging, theory and applications of transport in porous media, vol. 13. Dordrecht: Kluwer Academic Publishers; 1999.

[24] Kaviany M. Principles of heat transfer in porous media, mechanical engineering series. 2nd ed.. New York: Springer; 1999.

[25] Siegel R, Howell J. Thermal radiation heat transfer. 4th ed. New York: Taylor \& Francis; 2002.

[26] Modest MF. Radiative heat transfer. 2nd ed.. San Diego: Academic Press; 2003.

[27] Dombrovsky LA. Radiation heat transfer in disperse systems. New York: Begell House; 1995.

[28] Liu LH. Discrete curved ray-tracing method for radiative transfer in and absorbing-emitting semitransparent slab with variable spatial refractive index. Journal of Quantitative Spectroscopy and Radiative Transfer 2004;83:223-8.

[29] Liu LH, Zhang HC, Tan HP. Monte Carlo discrete curved ray-tracing method for radiative transfer in an absorbing-emitting semitransparent slab with variable spatial refractive index. Journal of Quantitative Spectroscopy and Radiative Transfer 2004;84:357-62.

[30] Petrasch J, Haussener S, Lipiński W. Discrete vs continuum level simulation of radiative transfer in semitransparent two-phase media. In: Webb B, Lemonnier D, editors. Proceedings of the 6th international symposium on radiative transfer RAD-10, Antalya; 2010. 